T9 wiederum integrierten die Normativität in ihre eigene Argumentation, man kann also eher von der Strategie „Inklusion“ sprechen. T7 begründet die Legitimität von Werten innerhalb der soziologischen Forschung durch die Naturwissenschaften. T9 konstruiert einen „Anti-Weber“, der in politische Aktivitäten involviert war und um die Unmöglichkeit absoluter Wertfreiheit wusste.

An der Analyse zu den Textabschnitten zur Wertneutralität der Soziologie wird die Doppelstruktur der Soziologie besonders deutlich: Alle Interviewabschnitte zeichneten sich durch eine besondere Legitimierungsrhetorik aus. Zudem gab es unterschiedliche Referenzfolien, mit der Normativität in der Soziologie umzugehen. Auch hier kam keine Einheitlichkeit in den Sinnkonstruktionen zum Vorschein, sondern die Kontingenz und Fluidität der Selbstbeschreibungen in Hinblick auf den adäquaten Umgang mit der Doppelstruktur der Soziologie.

\title{
4.2 DAS VERHÄLTNIS ZWISCHEN DER SOZIOLOGIE UND IHREM UNTERSUCHUNGSGEGENSTAND
}

In Abschnitt 4.1 haben wir bereits wesentliche Hinweise für eine Selbstbeschreibung der Soziolog(inn)en in Hinblick auf die Soziologie als Disziplin, ihre eigene Praxis wie auch auf mögliche Grenzziehungen zwischen der Soziologie und ihrem Untersuchungsgegenstand bekommen. Die Sichtweise, die dort untersucht wurde, war überwiegend auf Beschreibungen der Soziologie fokussiert. In Abschnitt 4.2 wird verstärkt das Verhältnis zwischen der Soziologie und ihrem Untersuchungsgegenstand in den Blick genommen, es geht also darum, wie Sozio$\log (\mathrm{inn})$ en das Verhältnis zwischen Soziologie und Nicht-Soziologie definieren.

\subsubsection{Der öffentliche Auftrag der Soziologie}

In den Interviews wurde unter anderem gefragt, ob die Interviewten einen „öffentlichen Auftrag“ der Soziologie bestimmen können. Diese Frage zielte auch auf Selbstbeschreibungen der Soziologie in Hinblick auf ihre Schnittstelle mit der Praxis ab. 


\title{
Analyse ausgewählter Interviewabschnitte
}

\author{
Interview T7, Zeile 254-297
}

254

255

256

258

259

260

261

262

263

264

265

266

267

268

269

270

271

272

273

274

275

276

277

278

279

280

281

282

283

284

285

286

287

288

I Hm Hm. Ähm gibt es denn in Ihrer-in Ihren Augen so was wie ein- einen öffentlichen Auftrag der Soziologie?

T7 Ja ich tu mich ein bisschen schwer damit mit dem- mit dem Auftrag, also ich- da hab' ich noch eh vielleicht auch ein engeres Wissenschaftsverständnis, wo ich dann sage okay, ja, natürlich die Wissenschaft kann nicht zu irgendwas beauftragt werden, äh Erkenntnisgewinne sind sozusagen und auch die Neugierde, ne wissenschaftiche Orientierung, die muss aus einem Interesse an der Sache heraus generiert äh werden und äh natürlich kann man sagen, wissenschaftlich öffentlich Finanziertes muss sich auch rechtfertigen, aber das ist natürlich auch ein bisschen ne Falle, die man sich dann äh stellt. Ich halte wenig von dieser Diskussion über ne öffentliche Wissenschaft, äh da ist ja jetzt immer auch viel äh in unserem Fachblättchen äh äh da gekommen. Ich halt' auch wenig von der-von dem Label kritische äh Wissenschaft. Ich glaube, wenn man gute Soziologie betreibt und sich auch aktiv irgendwie einbringt und engagiert, dann ist das beides Teil dessen, aber dieses Labelling äh ist etwas, was mich eher äh abschreckt, also ob etwas kritisch ist oder nicht, das ergibt sich äh aus dem Diskurs und aus-ausaus der Haltung, die man einnimmt, nicht unbedingt sozusagen aus der Marke, äh die man da erzeugen will, äh genauso glaub' ich, dass ne ganze Menge wichtige Einflüsse auf äh der Soziologie auf die Gesellschaft eben außerhalb dieser Marke öffen- Public Sociology laufen. [...] Also äh das is irgendwie ne Bewegung oder ein Schuh, den würd' ich mir nicht äh nicht anziehen, vielleicht muss ich das auch- muss ich das auch nicht und hab'das wär' auch nicht- auch nicht unbedingt äh nötig, aber ich seh' das eher als Krisenindikator äh für das Fach, wenn man solche Diskussionen äh aufmacht und äh irgendwie nich verzweifelt immer nach oben blicken oder so, ich bin ja auch noch da und ich hab' auch was zu sagen, //I: hm// äh würd' ich sagen jetzt, was so alles geschrieben wurde zu der Public, das sind ja alles Präliminarien,ne, also ich finde just do it, @mach's doch einfach@äh und redet nicht so viel darüber, dass man's machen sollte. Also wennmandie@Energieda rein@gegeben hätte mit interessanter Forschung, äh sich öffentlich sichtbar zu machen, ich glaub' dann hätte das Fach insgesamt viel mehr gewonnen als sozusagen äh ewig rekursive Diskurse darüber, dass man nun endlich mal Public Sociology werden möchte, aber das ist so bisschen meine private kritische Haltung. Ich weiß auch 
289

290

291

292

293

294

295

296

297

nicht, //I: ja// vielleicht tu ich da auch vielen Leuten äh äh Unrecht, äh die so was äh äh betreiben, aber ich würde mir generell wünschen, dass die Leute äh aktiver sind, das hat auch was ein bisschen mit Publikationsform und Formaten äh zu tun, auch ein bisschen mit der Fähigkeit, sich auszudrücken und äh in der Öffentlichkeit zu adressieren. Das sind ja auch ganz eigene äh Logiken und äh auch sozusagen Diskursarten, auf die man dann da trifft, die dann auch oft befremdlich sind und sicher auch missverstanden fühlt, äh aber das ist ja auch ne Frage, die man ein bisschen mit einüben äh kann und muss.

\section{Formulierende Feininterpretation}

Thema: Der öffentliche Auftrag der Wissenschaft (Z. 256-264)

T7 sieht die Frage nach einem ,öffentlichen Auftrag der Soziologie“ kritisch. Er bezieht sie auf die Wissenschaft im Allgemeinen und definiert in seinen Ausführungen dazu sein Wissenschaftsverständnis, das nicht mit einer Auftragsorientierung vereinbar ist. Stattdessen rekurriert er auf Erkenntnisgewinn und Neugierde als Triebfedern für die Wissenschaft. Er bezeichnet den Rechtfertigungsdruck der öffentlich finanzierten Wissenschaft als „Falle“.

Thema: Kritische und öffentliche Wissenschaft als Label (Z. 264-288)

In diesem Abschnitt geht T7 auf das „Labelling“ von Wissenschaft im kritischen oder im öffentlichen Sinn ein. Er äußert sich kritisch über das „Labelling“ der Soziologie als „Marke“. Er charakterisiert dies als selbstreferenziell und sieht es als Krisenindikator für das Fach. Vielmehr sollten die Forschenden gemäß dem Motto ,just do it“" öffentlichkeitswirksam werden.

Thema: Aktive Wissenschaft als öffentliche Wissenschaft (Z. 288-297)

Hier resümiert T7 seine eigene Einstellung. Er stellt dar, dass schon viel gewonnen wäre, wenn die Menschen sich aktiver in die öffentlichen Debatten einbringen würden. Hier definiert er Kriterien, die dafür notwendig wären: Publikationsformen, Formate und die „Fähigkeit, sich auszudrücken“.

\section{Reflektierende Interpretation}

Thema: Der öffentliche Auftrag der Wissenschaft (Z. 256-264):

Argumentation mit dem Hintergrundmodus einer Bewertung

T7 betreibt in diesem Abschnitt Grenzarbeit zu dem Begriff eines „öffentlichen Auftrags der Soziologie“. Er nutzt diese Grenzarbeit zu einer Selbstbeschreibung: „da hab' ich [...] vielleicht auch ein engeres Wissenschaftsverständnis“. Soziologie, die er in diesem Abschnitt der Wissenschaft zuordnet, muss Krite- 
rien einer klassisch wissenschaftlichen Grenzziehung entsprechen, wie wir sie bereits in Kapitel 2.3.3 gehört haben: Erkenntnisgewinn und Neugierde im Gegensatz zu externer Zweckorientierung - science for science sake in dem Sinn, als das wissenschaftliche Interesse aus einem „Interesse an der Sache heraus generiert“ werden muss. Diese Grenzarbeit kann im Sinne einer ,protection“ der wissenschaftlichen Autonomie gegenüber wissenschaftsexternen Ansprüchen interpretiert werden. Zwar formuliert er ebenso das Gegenargument einer ,öffentlich“ finanzierten Wissenschaft, die sich „auch rechtfertigen“ muss, definiert dies aber sofort als eine „Falle“.

Thema: Kritische und öffentliche Wissenschaft als Label (Z. 264-288):

Argumentation mit dem Hintergrundmodus einer Bewertung

In diesem Abschnitt betreibt T7 Grenzarbeit zu unterschiedlichen „Labels“, die der Soziologie mit Blick auf ihren öffentlichen Auftrag gegeben werden. Das eine Label ist die kritische Wissenschaft, das andere das der public sociology. So macht er seine Position deutlich, die der von T9 entgegensteht, der public sociology und damit ihren Vertreter Michael Burawoy zu einer Referenzfolie der Selbstbeschreibung nutzt (siehe 4.1.4.2). T7 entwickelt vielmehr seine eigene Referenz, ein „mach's doch einfach“, und ruft zu einem pragmatischen Umgang mit öffentlicher Soziologie auf, die keine Labels oder Vorgaben braucht.

Thema: Aktive Wissenschaft als öffentliche Wissenschaft (Z. 288-297):

Argumentation mit dem Hintergrundmodus einer Bewertung

Im letzten Abschnitt geht T7 auf die Logik der Praxis ein und betreibt hier wiederum Grenzarbeit. Zunächst äußert er seinen Wunsch, dass die Forschenden aktiver sein sollten. Hier wiederholt sich der Autonomiegedanke aus dem ersten Abschnitt. Statt für einen ,von außen“ vorgegebenen Auftrag der Soziologie plädiert T7 für ein Interesse an der Praxis, das aus den Forschenden heraus entsteht, ohne disziplinäre Labels und öffentliche Auftraggeber. Die NichtSoziologen, die er zunächst mit „Öffentlichkeit“ beschreibt, können aus seiner Sicht am besten über adressatengerechte Publikationsformen und Formate angesprochen werden. Hier grenzt er die Soziologie jedoch nicht nur über unterschiedliche Kommunikationsformen von der Nicht-Soziologie ab. Ebenfalls zeichnet er Soziologie und Öffentlichkeit als getrennte Welten, die über eigene Logiken und Diskursarten verfügen. Diese sind für die jeweils andere Partei ,,befremdlich“ und bergen die Gefahr, dass man sich „missverstanden fühlt". Der Umgang mit der Öffentlichkeit muss erst erlernt und eingeübt werden. Soziologisches Wissen unterscheidet sich in diesem Zusammenhang also grundlegend von anderen Wissensformen. 
Interview T8, Zeile 240-300

240 I Hm. Gibt es denn Ihrer Meinung nach einen öffentlichen Auftrag der Sozio$241 \quad$ logie?

242 T8 Ja natürlich

$243 \mathrm{I} \quad \mathrm{L}_{j a}$

244 T8 ein- eindeutig. Es gibt einen öffentlichen Auftrag der Soziologie, im Grunde $245 \quad L e b e n s w e i s e n$ quasi der-dem gesellschaftlichen Publikum insgesamt, ähm 246 wie soll ich sagen, untereinander verstehbar zu machen, also die-diesen Übersetzungsprozess von gesellschaftlicher Wirklichkei-von gesellschaftlichen Wirklichkeiten für eine gesellschaftliche Öffentlichkeit deutlich zu machen. damit hab' ich einen relativ traditionellen Begriff der Bedeutung von Öffentlichkeit. also ich bin-das ist sozusagen so ein 19. Jahrhundert-Begriff von Öffentlichkeit, den ich immer nach wie vor habe. ich glaube immer noch, es gibt gesellschaftliche Öffentlichkeit und die ist eine, etwas, was die Leute interessiert, //I: hm// also irgendwie diese ähm sozusagen die- derder Streit darum, was uns gemeinsam angeht und wie wir das Leben interpretieren aus unterschiedlichen Perspektiven. das ist sozusagen Soziologie und das ist eigentlich glaub' ich der Auftrag von Soziologie, //I: hm// also der öffentliche Auftrag von Soziologie besteht darin, den gesellschaftlichen Selbstübersetzungsprozess in Gang zu halten, //I: hm// aber ihn gleichzeitig auszurichten an einer Idee von kollektiver Handlungsfähigkeit. Hm. Gibt es denn etwas, was die Soziologie ähm also wie sie nicht wirksam werden sollte aus Ihrer Sicht?

T8 Na die Soziologie sollte auf keinen Fall, das ist mein Problem ein bisschen mit der Generation der jetzt Vierzigjährigen in der Soziologie, Soziologie darf sich nicht auf eine, ja die Gefahr der Soziologie besteht darin, dass sie sich auf eine Art von ähm Konzeptwissenschaft oder Ansatzwissenschaft reduziert, also dass sozusagen-also ich merke immer mehr, dass ich in den-in der- bei den jetzt Vierzig- End-Vierzigjährigen auf Kolleginnen und Kollegen treffe, die einen Ansatz befolgen oder gar vertreten Rational Choice oder irgendwie Konflikttheorie oder Systemtheorie oder irgend so was //I: hm// und das hab' ich immer für merkwürdig gehalten. Also diese Idee der Selbstverschanzung am Ansatz, ohne sich zu fragen, was bedeutet eigentlich dieser Ansatz und was will ich damit eigentlich sagen, auf welche Arten von Problemen und welche Phänomene reagiert eigentlich dieser Ansatz. das halte ich für ne große Gefahr für die Soziologie, //I: hm// also eine Art, also ich bin-finde es sehr gut, dass es einen Professionsbegriff der Soziologie gibt, ich glaube, ich würde auch einiges dafür tun, diesen Professi- 
onsbegriff von Soziologie zu stärken ähm ich glaube die Soziologie kann sich darüber und wird sich mehr und mehr als Profession konsolidieren können, //I: hm// das muss sie auch glaub' ich, nicht nur methodisch. das ist immer die Idee, sie kann sich nur methodisch konsolidieren. das ist glaub' ich ein Fehl- ist ein Fehler quasi, sie muss sich auch begrifflich konsolidieren und ähm und wenn man das- wenn man das sozusagen sicher weiß, dann kann man auch die Öffent- die Öffentlichkeitsfunktion von Soziologie wahrnehmen. Sie brauchen eine Sicherheit in der Konventionalität der Disziplin //I: hm// dann können Sie auch quasi der Öffentlichkeit sagen, so jetzt sag' ich Ihnen mal, was ein Soziologe dazu sagen wird, denn das ist meine Erfahrung immer wieder gewesen, dass die Leute gesagt haben, ich weiß, was ein Ökonome sagt, ich weiß, was ein- eine Psychologin sagt und ich weiß auch, was ein Pädagoge sagt usw., ich will jetzt mal wissen, was ne Soziologin oder ein Soziologe dazu sagt und das ist glaub' ich wichtig, die Sicherheit zu haben. jetzt sage ich als Soziologe etwas dazu //I: hm// und ich kann auch etwas dazu sagen, und das ist sozusagen diese Doppelheit und das, wenn die Soziologie das verliert, also //I: hm// die Sicherheit in ihrer Professionalität und Disziplinarität und den-den-auch den Mut, sich dann quasi in der öffentlichen- im öffentlichen Intellektualität zu positionieren, dann ist es glaub' ich ähm- ist es- die öffentliche Finanzierung ist sozusagen die Soziologie nicht mehr wert //I: hm hm// das ist ja ne alimentierte Wissenschaft und die Öffent- die Öffentlichkeit muss irgendwo auch ein Gefühl dafür kriegen, dass man mit der Sozio-dass sie zu irgendwas Sinn hat und nicht nur in ihrer eigenen Professionalität ihren Sinn findet.

\section{Formulierende Feininterpretation}

Thema: Der öffentliche Auftrag der Soziologie (Z. 240-259)

Für T8 besteht ein öffentlicher Auftrag der Soziologie. Er definiert in diesem Zusammenhang seinen Begriff von Öffentlichkeit. Den öffentlichen Auftrag der Soziologie sieht er darin, „den gesellschaftlichen Selbstübersetzungsprozess in Gang zu halten, aber ihn gleichzeitig auszurichten an einer Idee von kollektiver Handlungsfähigkeit"“. 
Thema: Der Professionsbegriff der Soziologie (Z. 262-286)

Auf Nachfrage, wie denn die Soziologie nicht wirksam werden sollte, macht T8 eine interessante Abgrenzung zur eigenen Disziplin. Er sieht die Soziologie in Gefahr, zu einer reinen Konzeptwissenschaft zu werden. Wichtig ist ihm deshalb ein Professionsbegriff der Soziologie: Diese muss sich als (öffentlichkeitswirksame) Profession methodisch und begrifflich konsolidieren.

\section{Thema: Notwendigkeit der Konventionalität der Disziplin (Z. 286-300)}

Diese Konsolidierung mündet in eine „Sicherheit in der Konventionalität der Disziplin", damit sie ihre Öffentlichkeitsfunktion wahrnehmen kann. T8 zieht Vergleiche zur Ökonomie, zur Psychologie und zur Pädagogik, die bereits über solch eine Sicherheit verfügen. Zusätzlich dazu braucht die Soziologie „Mut“, sich in der „öffentlichen Intellektualität“ zu positionieren. Diese Kriterien verbindet er mit der öffentlichen Finanzierung der Soziologie, die er als alimentierte Wissenschaft bezeichnet. Sie steht unter einem Legitimierungsdruck, denn sie soll zeigen, dass sie „zu irgendetwas Sinn hat“ und nicht rein selbstreferenziell arbeitet.

\section{Reflektierende Interpretation}

Thema: Der öffentliche Auftrag der Soziologie (Z. 240-259):

Argumentation mit dem Hintergrundmodus einer Bewertung

Im Gegensatz zu T7 stellt T8 einen öffentlichen Auftrag für die Soziologie fest. Für ihn geht es hier weniger darum, die Autonomie der Soziologie zu verteidigen, als darum, eine Aufgabe der Soziologie in der Öffentlichkeit zu definieren. Er ist überzeugt, dass soziologische Expertise anderen Wissensformen überlegen ist, da sie eine Mittlerfunktion zwischen ,gesellschaftlichen Wirklichkeiten“ erfüllt. Er markiert dies rhetorisch als Übersetzungsprozess gesellschaftlicher Wirklichkeiten bzw. als Möglichkeit, das gesellschaftliche Publikum „untereinander verstehbar zu machen“. Hier ist interessant, dass er die Öffentlichkeit als passive Masse, als „Publikum“ definiert, das letztlich auf die Soziologie angewiesen ist. Er bleibt bei seiner Definition des Begriffs von Öffentlichkeit auf der epistemisch-kognitiven Ebene. So umkreist er wiederum die Definition der Aufgabe der Soziologie, indem er sie als Streit darum beschreibt, wie „wir das Leben interpretieren aus unterschiedlichen Perspektiven". In einem Abschlussresümee geht er schließlich noch auf den Handlungsbegriff ein und erweitert seine ursprüngliche Definition der Aufgabe der Soziologie als ,gesellschaftlichen Selbstübersetzungsprozess“ um die Idee der „kollektiven Handlungsfähigkeit“. Soziologie soll also nicht nur einen „gesellschaftlichen Selbstübersetzungsprozess in Gang halten“, sondern diesen zusätzlich an einer „Idee von kollektiver 
Handlungsfähigkeit" ausrichten. Auch diese Aussage bleibt auf der epistemischkognitiven Ebene und orientiert sich an einer Selbstbeschreibung, die auf einem theoretisch-soziologischem Vokabular basiert.

\section{Thema: Der Professionsbegriff der Soziologie (Z. 262-286):}

\section{Beschreibung mit dem Hintergrundmodus einer Bewertung}

Die Eingangsfrage nach dem öffentlichen Auftrag der Soziologie gab viel Raum zur Abgrenzung. Abgegrenzt hat sich T8 jedoch nicht von einer bestimmten Art von Öffentlichkeitswirksamkeit der Soziologie, sondern von den eigenen Fachkollegen. Er definiert die „Gefahr“, dass die Soziologie zu selbstreferenziell wird - er bezeichnet dies als Ansatz der Selbstverschanzung. Er sieht die Reichweite der Soziologie in die Gesellschaft hinein, wofür er auf soziologisches Vokabular zurückgreift, nämlich auf den Professionsbegriff. Der Klient der Soziologie ist die Gesellschaft. Hier wird sein Verständnis von Soziologie klar, das in gewisser Hinsicht von dem von T7 abweicht. Zwar haben beide die Absicht, etwas in der Gesellschaft zu verändern. T7 sieht dies jedoch stärker als individuelles Unterfangen und grenzt sich von jeglichem gemeinsamen „Labelling“ ab, das er als zu selbstreferenziell abwertet. T8 sieht den umgekehrten Weg, eine methodische und begriffliche Konsolidierung, um überhaupt in der Lage zu sein, die Öffentlichkeit zu erreichen.

Thema: Notwendigkeit der Konventionalität der Disziplin (Z. 286-300):

\section{Argumentation mit dem Hintergrundmodus einer Bewertung}

Dass eine Konventionalität der Disziplin notwendig ist, kann auch mit dem Sprechen mit einer Stimme interpretiert werden. Vergleichshorizonte sind andere Disziplinen wie die Ökonomie oder die Psychologie, die diese Konventionalität bereits erreicht haben. Es geht hier um eine Fremdbeschreibung der Soziologie, um die Sicherheit zu wissen, was denn „ein Soziologe“ dazu sagen könnte. Dann eröffnet T8 quasi ein dialektisches Verhältnis in der Doppelrolle der Soziologie: zwischen ihrer Professionalität und ihrer Disziplinarität. Sollte sie diese Doppelrolle verlieren, ist sie nach T8 auch die öffentliche Finanzierung nicht mehr wert. Hier wird auch ein Kontrast zu T7 deutlich, der gerade den Diskurs um die öffentliche Finanzierung als ,Falle“ beschreibt.

\section{Orientierungsmuster „Der öffentliche Auftrag der Soziologie“}

\section{Autonomie der Soziologie}

Die beiden Interviewten haben unterschiedlich auf die Frage nach einem öffentlichen Auftrag der Soziologie reagiert. T7 hat sich von einem öffentlichen Auf- 
trag abgegrenzt, da er die Autonomie der Wissenschaft im Sinne einer „Grenzabsicherung" (protection) verteidigen wollte. Wissenschaft wird in der Selbstbeschreibung als intrinsisch interessengeleitet und nach Erkenntnis strebend konzipiert und nicht als etwas, das von einem externen Auftraggeber beeinflusst wird. Dementsprechend kritisch sieht T7 auch eine öffentliche Soziologie, die sich den Steuerzahlern gegenüber in einer Legitimierungspflicht sieht.

T8 konzipiert eine ganz andere Rhetorik um die öffentliche Aufgabe der Soziologie. Der Autonomiegedanke ist quasi der Antwort bereits inhärent. Soziologie wird hier als eine Wissensart entworfen, die der Gesellschaft überlegen und in der Lage ist, notwendige gesellschaftliche Selbstübersetzungsprozesse anzuregen, damit eine kollektive Handlungsfähigkeit entsteht. Dies zeigt sich bereits in der Art und Weise, wie T8 diese Gedanken äußert: Er verbleibt im Begriffsvokabular der Soziologie. Er stellt einen anderen Bezug zu der Legitimierungspflicht der Soziologie als alimentierte Wissenschaft her als T7 und in diesem Sinn schränkt T8 ihre Autonomie auch wieder ein: Sie macht nur Sinn, wenn sie auch öffentlich relevante Themen und nicht nur sich selbst adressiert.

\section{Soziologie als der Gesellschaft überlegenes Wissen}

Obwohl sich T7 von einem wissenschaftspolitisch induzierten öffentlichen Auftrag der Soziologie abgrenzt, äußert er doch an anderer Stelle eine Motivation, die er als Auftrag der Soziologie beschreibt:

„Ja das seh' ich schon, also so bisschen normative äh Aspekte spielen natürlich schon äh mit rein ( ) schon um Projekte, gesellschaftlichen, also @Optimierung will@ ich nicht sagen, aber Selbstverbesserung, äh auch vor dem Hintergrund relativ abstrakter äh Vorstellungen wie Menschenwürde, äh Gleichheit, Solidarität, dass das durchaus was ist, wo ich äh sage, das ist äh- das ist mir äh wichtig und dafür würd' ich da, ich sag' mal, dafür betreib' ich ja auch ein bisschen äh Soziologie, weil mir auch das Gesellschaftliche und irgendwie auch das Fortkommen der Gesellschaft äh mir am Herzen äh liegen. ein anderer Aspekt ist natürlich immer, ja diese, was ich vorhin schon sagte, diese Neugierde oder $\mathrm{Cu}$ riosity, also äh wo man sagt, das ist auch etwas, was- was einen irgendwie- was einen irgendwie trägt und das hat man auch nicht- auch nicht für alle- für alle Themen, äh von daher seh' ich schon, also jetzt nicht im Sinne eines gesellschaftlichen Auftrages, aber doch äh durchaus ein Anliegen, was auch mit der Soziologie äh verbunden ist und was sich auch auf bestimmte soziologische Denktraditionen äh durchaus äh berufen kann. ein anderer Aspekt, der jetzt auch mit der Aufklärung äh zu tun hat, also Aufklärung als Selbst- äh Selbsterkenntnis, äh klar, ich hab' vorhin so biss- bisschen abgewertet mit der kritischen Soziologie, aber sozusagen die- die Immanenz von Kri- Kritik ist für mich auch äh sozusagen Teil- Teil der Soziologie, auch Immanenz sozusagen der Befähigung zur Kritik von 
äh von Gesellschaft, also Reflexion im Hinblick auf äh ja Entwicklungen, Tendenzen, Strukturveränderung vor dem äh Hintergrund so bisschen abstrakterer Ziele, aber Aufklärung auch im Sinne von Mythenjäger zu sein.“ (T7, Z. 985-1004)

Durch diese Interviewpassage wird die Ambivalenz deutlich, die bei T7 in Hinblick auf einen öffentlichen Auftrag der Soziologie besteht. Denn hier äußert er durchaus ein Interesse an gesellschaftlicher Veränderung, diese darf aber nur aus intrinsischem Interesse und nicht aus Zwang heraus entstehen. Trotz ihrer unterschiedlichen Selbstbeschreibungen im Hinblick auf den öffentlichen Auftrag der Soziologie bestehen folglich Gemeinsamkeiten bei den beiden Interviewpartnern: Beide gehen darauf ein, dass die Soziologie eine öffentlich wirksame Wissenschaft sein muss. Dies geschieht, indem sie sich in der Selbstbeschreibung als Soziologen konzipieren, die der Gesellschaft überlegenes Wissen produzieren und Prozesse der „Selbstverbesserung“ oder „Selbstübersetzung“ anregen. Soziologie stellt somit quasi Hilfe zur Selbsthilfe dar. In diesem Sinn distanziert sich T7 auch wieder von seiner abwertenden Aussage zum „Label kritische Soziologie“, das er nun selbst mit Inhalt füllt.

In beiden Fällen findet eine Grenzziehung zwischen der Soziologie und der „Öffentlichkeit“" statt, die sich durch unterschiedliche Logiken auszeichnet. Soziologie kann distinguiertes Wissen in den öffentlichen Diskurs einbringen. Die Selbstbeschreibung von T7 als „Mythenjäger“ steht sinnbildlich dafür.

\section{Individualismus vs. Konsolidierung}

Die Wege, auf denen T7 und T8 eine öffentlich relevante Soziologie einfordern, sind jedoch unterschiedlich. Die Selbstbeschreibung, der T7 folgt, kann als Individualismus bezeichnet werden. Hier geht es überwiegend um die individuelle Verantwortung, interessante Forschung in die öffentliche Diskussion einzubringen und unterschiedliche Kommunikationsformate zu erlernen. Im Gegensatz dazu prägt T8 das Bild der disziplinären Konsolidierung und des Sprechens mit einer Stimme. In diesem Zusammenhang verwendet er den Begriff der Profession, der es ihm erlaubt, eine in sich wiederum begrifflich konsolidierte Schnittstelle zwischen der Disziplin und ihrem Untersuchungsgegenstand herzustellen. Wie bereits in 4.1.3 sehen wir auch hier, dass unterschiedliche Selbstbeschreibungen aufeinandertreffen, die einerseits auf die Soziologie als Ganzes und andererseits auf das Individuum eingehen. 


\subsubsection{Verantwortung gegenüber der Praxis}

Im Interviewverlauf wurde deutlich, dass die Frage der Verantwortung eine große Rolle spielte. Diese wurde in divergente Richtungen interpretiert. Die folgenden Abschnitte zeigen unterschiedliche Aussagen zur Verantwortung gegenüber der Praxis. Ausschlaggebend für die Auswahl der Interviewabschnitte war nicht die Fragestellung, sondern die Thematisierung von Verantwortung in den Antworten.

\section{Analyse ausgewählter Interviewabschnitte}

Interview T6, Zeile 180-222

180 T6 Ich hab mal vor ganz ganz vielen Jahren äh ein Projekt gemacht, [...] also ganz am Anfang und äh der Mensch vom Ministerium hat g'sagt, wissen Sie, am Ende bräucht ich haltwas, was ich bauen kann@(.)@.

183 I @Okay@.

184 T6 Ja, da han ich g'sagt, ich-ich-ich-Sie können mit dem machen, was Sie wollen, aber ich denk mir nicht aus, was Sie tun. //I: hm// also es ist ein bisschen äh ne Haltung und das seh ich auch selber, äh wie die Leute, die die Atombombe erfunden haben, //I: hm// ja die haben g'sagt äh wir-so geht das, ja, so, was ihr damit anfangt, das ist die Frage, ja, also macht sie das moralisch schuldig, ist man verantwortlich, wenn man die Atombombe erfindet, äh dafür, was dann damit angestellt wird oder geht's um eine Problemlösung. //I: hm// und ich seh das, also ich bin da schon wirklich äh ich beobacht' mich da auch selber und sag klar, du-du en- sozusagen $d u$ nimmst deine- einfach bestimmte Art von Verantwortung nicht an, tu ich nicht, ja, also nicht als Soziologe. wenn ich nicht als Soziologe agiere, dann äh bin ich ein heftig politischer und moralisch nicht-vielleicht moralisch manchmal merkwürdiger Mensch, aber ich hab da natürlich viel Moral, ja, aber wenn ich Soziologie betreib', das ist nicht so, dass ich keine Moral hab', aber ich versuch' die Moral derer zu übernehmen, mit denen ich mich beschäftige //I: hm// und das ist ein gewaltiger Unterschied, ja und deshalb sagen wir auch, wir alle können diese Art von Soziologie, von Forschung nicht mit jedem Thema machen, man muss sich das vorher klarmachen, ja. äh ich muss schon wissen, ja also entweder muss ich sagen okay, man kann ja auch anders forschen, man muss sich nicht auf jetzt äh auf-auf alles einlassen und sagen, ich mach da mit, //I: ja,// ja, aber unser- unsere Grundidee ist, wir gehen hin und- und sind-werden solche wie die, die wir untersu- 
206

207

208

209

210

211

212

213

214

215

216

217

218

219

220

221

222

chen, aber da muss man wissen, ja, also wenn ich dann sag okay, ohne zu untersuchen, ich weiß-weiß ich, Neonazis, ja //I: hm hm// äh dann- danndann nicht mit der Haltung äh das sind aber merkwürdige Leute, sondern da muss ich Neonazi werden, ja und da //I: hm, okay// sozusagen die moralische Entscheidung liegt eigentlich vorher zu sagen, ich untersuch' das halt nicht mit den Mitteln, ja, //I: hm hm// Sie können mir einen Auftrag erteilen, dann mach' ich da ne distanzierte Form von Forschung, das mach'ich ja auch, aber wenn Sie mich danach fragen, wie ich gern arbeite, ja, dann //I: hm, ja, ja, ja// ist das genau dieses und deshalb findet eigentlich die moralische Entscheidung vorher statt, ja, und dann sag ich gut, so, und wenn ich sag, ich guck mir jetzt egal was an, ja, dann versuch'ich maximal die nicht mehr so fremd zu finden, sondern ganz im Gegenteil. [...] so, also sich einlassen bedeutet für mich dann wirklich die Perspektive, die Moral oder was auch immer derer zu übernehmen, mit denen ich zu tun hab'. so und wenn man sagt, aber mit denen will ich nichts zu tun haben, sollte man halt die Art Forschung, deshalb kann man die ja trotzdem erforschen //I: hm hm,// aber dann macht man was anderes, $j a$.

\section{Formulierende Feininterpretation}

Thema: Verantwortung und Forschung (Z. 180-194)

T6 beschreibt an einem Beispiel, wie er Verantwortung in der Forschung sieht. Er weist die Übernahme von Verantwortung für seine Forschungsergebnisse zurück und konkretisiert seinen Standpunkt, indem er den Vergleich zu der Entwicklung der Atombombe zieht.

\section{Thema: Moral und Forschung (Z. 194-222)}

T6 geht dann auf seine moralische Gesinnung ein und spricht über Moral in der Forschung. Er verdeutlicht seinen Forschungsansatz, für den besonders relevant ist, dass der Forscher „die Moral derer“ übernimmt, die er untersucht. Deshalb wäre für ihn eine Untersuchung von Neonazis schwierig, da er zum Neonazi werden müsste. Er erläutert, dass die moralische Entscheidung also bereits in der Wahl des Untersuchungsobjekts liegt.

\section{Reflektierende Feininterpretation}

Thema: Verantwortung und Forschung (Z. 180-194):

Erzählung mit dem Hintergrundmodus einer Argumentation

Zunächst leitet T6 seine Argumentation mit einer Erzählung ein, in der er den zeitlichen Abstand zu dem Projekt betont, das er für die Beantwortung der Frage anführt. Er beendet diese kurze Erzählung damit, dass er die Soziologie von jeg- 
licher Handlungsorientierung abgrenzt: Wie die Forschung verwendet wird, interessiert den Forschenden nicht. In seiner Argumentation schwenkt er nun über zu der Frage der moralischen Schuld. Um seinen Standpunkt zu veranschaulichen, zieht er einen starken Vergleich: zur Erfindung der Atombombe. Das ist in diesem Zusammenhang ein starkes Bild, weil T6 in seinem Vergleich implizit die Reichweite der Soziologie mit der Erfindung und Anwendung der Atombombe gleichsetzt und mit der Schuldfrage verbindet, wer für die verheerenden Folgen des Einsatzes der Bombe verantwortlich ist. T6 postuliert, dass er als Soziologe „eine bestimmte Art von Verantwortung" für seine Forschung nicht annimmt. Die Grenze zwischen der Soziologie und ihrem Untersuchungsgegenstand wird also scharf gezogen, auch, was mögliche Folgen der Forschung angeht.

\section{Thema: Moral und Forschung (Z. 194-222):}

\section{Beschreibung mit dem Hintergrundmodus einer Argumentation}

Dennoch scheint T6 das Thema der Moral stark zu beschäftigen. Im weiteren Verlauf seiner Argumentation gerät er entsprechend in die Defensive. Er zieht eine Grenze zwischen dem Agieren als Soziologe und dem Agieren als NichtSoziologe, was auf eine Rollentrennung hinausläuft. Als Nicht-Soziologe beschreibt er sich als ,heftig politisch und moralisch“. Die Vergewisserung seines moralischen Ichs als Nicht-Soziologe nimmt viel Raum ein. Seine eigene Moral unterscheidet sich von seiner Forschungsmoral: Er übernimmt die Moral derjenigen, die er beforscht. Er schlüpft also quasi in die Haut seiner Forschungssubjekte und blendet sich selbst als moralisch und politisch denkenden Menschen aus. Das stellt eine Distanzierung von eigenen normativen Vorstellungen dar, die er über eine Rollentrennung (Soziologe vs. Nicht-Soziologe) zu lösen versucht. Indem er bereits im Vorfeld eine Auswahl darüber trifft, wen er mit seiner Methode beforschen möchte und kann und wen nicht, verknüpft T6 den Begriff der Forschungsmoral mit der Entscheidung über den Gegenstand, die der Forschende bereits vor Beginn der Forschung fällt.

\section{Interview T5, Zeile 273-296}

273 I Was ist denn äh aus deiner Sicht Wissenstransfer? Wie würdest du das defi$274 \quad$ nieren?

275 T5 Das, was ich herausgefunden habe, ahm anderen Leuten mitzuteilen. und 276 Wissenstransfer kann ahm-also breit definiert ist das auch innerhalb der

277 Scientific Community, einfach sagen, ich weiß was, das ist toll und spannend $278 \quad$ und das möchte ich jetzt anderen auch erzählen. im engeren Sinne ist es in 279 der Tat so, dass ich mir einen Adressatenkreis überlege, die von dem, profi- 

ergelder ausgebe und damit auch ne gewisse (1) Verantwortung oder (2) nen Legitimations(2) ((schnalzt mit der Zunge)) -druck vielleicht habe oder vielleicht nicht unbedingt Legitimationsdruck aber einen gewissen- eine gewisse Verpflichtung, das ist das bessere Wort dafür //I: hm// ahm, das ich dass, was ich rausgefunden habe, dann auch tatsächlich denjenigen, die es betrifft auch mitteilen //I: hm// oder oder in eine Form gießen, die sie dann auch in der Tat akzeptieren können. //I: hm// also es bringt nichts zu sagen, lies meinen keine Ahnung was, Kölner Zeitschrift-Artikel, sondern dass man das eben so aufbereitet und das gehört für mich son Stück weit dazu, das war nich (3) nicht immer so, und ich weiß, das das machen auch nich alle Wissenschaftsinstitute so, das is, das nehme ich so wahr, das is eher am [Name der Organisation] das Credo ist, dass man sagt, wenn man problemorientierte Grundlagenforschung macht, dann muss man das auch irgendwann den den jeweiligen Betroffenen mit- oder mittteilen können. insofern empfind ich das auch $n$ Stück weit Verpflichtung, dass wir, die wir ja so gut ausgestattet sind, eben das dann auch machen.

\section{Formulierende Feininterpretation}

Thema: Verantwortung und Forschung (Z. 275-296)

Über die Frage nach dem Thema Wissenstransfer kommt T5 auf das Thema Verantwortung zu sprechen. Er thematisiert Verantwortung in Hinblick auf die Verpflichtung, Forschungsergebnisse den Betroffenen mitzuteilen.

\section{Reflektierende Feininterpretation}

Thema: Verantwortung und Forschung (Z. 275-296):

Beschreibung mit dem Hintergrundmodus einer Argumentation Im Gegensatz zu T6 sieht T5 eine Verantwortung gegenüber den „Adressaten“ der Forschung. Als er das beschreibt, zeigt er sich allerdings etwas zögerlich in seiner Rhetorik. Er sucht nach einer geeigneten Definition von Verantwortung, die er als Verpflichtung begreift. Er bezieht sich auf die Organisation, in der er arbeitet und mit der er sein Engagement begründet. Explizit weist er darauf hin, dass dies noch nicht immer seine Überzeugung war, die Organisation letztlich jedoch eine entscheidende Rolle gespielt hat. Die Beziehung zwischen Soziologie und Untersuchungsgegenstand wird in diesem Abschnitt nicht wie bei T6 problematisiert, jedoch wird das Verhältnis der Soziologie zu ihrem Untersuchungsgegenstand als ein einseitiges konzipiert, in dem die „Adressaten“ oder „Betroffenen“ Wissensrezipienten sind. 


\section{Interview T2, Zeile 555-574}

555 T2 Also natürlich gehört das dazu, einer wie auch immer gearteten Öffentlich-

$556 \quad k e i t, j a$, ob das eine Medienöffentlichkeit is oder es im privaten Kreis ver$557 \quad$ ständig zu machen, was man tut, das hat auch was mit Legitimation zu tun. 558 das ist äußerst schwierig zu legitimieren, dass man hier so abgefahrene Sachen macht, ja, äh äh äh in der Wissenschaft und keiner versteht mehr den Sinn, inklusive derjenigen äh demjenigen, die es betreibt, ja? äh äh, aber ma muss glaub ich auch anders rum mal agieren, ja, das is ganz schwierig, is glaube ich auch ein Legitimationsproblem. äh äh, ma muss auch vielleicht mal den den Mut haben nich nur zu sagen, was es denn nich alles noch bringen könnte an Reflexionswissen und sowas, ja? oder gar ah ah an technischen Vorprodukten, ja? sondern auch das, was wir nicht können und das was wir nicht wollen, wofür wir nicht da sind. (3) also mir scheint auchweil sonst hat ma son Anspruchsexpanionszirkel da drin, also da werden irgendwie Erwartungen gestellt, die man eh nicht leisten kann, dann dann fühlt man sich selber bedrängt, was man nich alles machen muss, ja? und dann kriegt mas- kriegt ma sozusagen an Kopf geschlagen, weil man jetzt ja gar keine Lösung entwickelt hat oder sowas (3), ja? also ich hm vielleicht äh darf ma muss ma eher so größere Offenheit, ja, äh was man denn macht und was man nicht macht: und unter dieser Bedingung nehm ich kein Geld. @(.)@.zum Beispiel.

\section{Formulierende Feininterpretation}

Thema: Legitimation von Wissenschaft (Z. 555-560)

In diesem Abschnitt stellt T2 heraus, dass die Kommunikation mit einer Öffentlichkeit „dazugehört“. Diese ist aus Legitimierungsgründen notwendig. Die Wissenschaft muss sich laut T2 letztlich immer legitimieren.

Thema: Grenzziehung zu Ansprüchen auf Anwendbarkeit (Z. 560-574)

In diesem Abschnitt beschreibt T2 die Notwendigkeit, Ansprüche gegenüber der Wissenschaft abzuwehren. Es geht also nicht darum, Versprechungen in Bezug auf die Leistungsfähigkeit der Wissenschaft zu machen, sondern darum, Position zu beziehen und aufzuzeigen, was eben nicht möglich ist. 


\section{Reflektierende Feininterpretation}

Thema: Legitimation von Wissenschaft (Z. 555-560):

Argumentation mit dem Hintergrundmodus einer Bewertung

Rhetorisch argumentiert T2 zunächst ähnlich wie T5, dass die Kommunikation mit der Öffentlichkeit zu den Aufgaben der Soziologie gehört. Er argumentiert jedoch ausschließlich mit der Begründung, dass man eine Legitimationspflicht habe und verständlich machen müsse, was in der Wissenschaft passiert.

Thema: Grenzziehung zu Ansprüchen auf Anwendbarkeit (Z. 560-574):

Bewertung mit dem Hintergrundmodus einer Argumentation

Im zweiten Abschnitt geht er dann auf Distanz zu dieser Legitimationspflicht. Er argumentiert nun in die andere Richtung: Es sei vielmehr Aufgabe darzustellen, was eben nicht machbar ist. In dieser Hinsicht geht er noch weiter als T6, indem er nicht nur eine Grenze zwischen der Wissenschaft und den Forderungen zieht, dass Forschung anwendbar sein soll, sondern indem er diese Grenze aktiv auszuweiten versucht. Rhetorisch setzt er starke Begrifflichkeiten ein, etwa „Anspruchsexpansionszirkel“" oder das Gefühl der Bedrängnis. Er nennt als Beispiel sogar die Möglichkeit, Gelder abzuweisen, die eine Anwendbarkeit von Forschung fordern. Obwohl in diesem Interviewabschnitt der Begriff der Verantwortung nicht explizit fällt, ist dieser doch der Argumentation implizit. T6 sieht es als seine Verantwortung gegenüber dem wissenschaftlichen Arbeiten an, Grenzen in Hinblick auf überhöhte Ansprüche an die Wissenschaft zu ziehen.

\section{Orientierungsmuster „Verantwortung gegenüber der Praxis“}

In den drei Interviewabschnitten wurde das Thema „Verantwortung“ in den Selbstbeschreibungen unterschiedlich eingesetzt. In allen Fällen markierte es eine Grenze bzw. Grenzüberschreitung zwischen der Soziologie und ihrem Untersuchungsgegenstand.

Während für T6 Verantwortung gegenüber der Praxis ein Demarkationsinstrument zwischen Wissenschaft und Praxis war (er ist „Verantwortungsverweigerer"), stellt Verantwortung für T5 eine Brücke zwischen Soziologie und ihrem Untersuchungsgegenstand dar. Diese entsteht aus einer Verpflichtung gegenüber der Praxis, die sich aus der öffentlichen Finanzierung der Institution begründet. Während T6 also den Schwerpunkt auf die Autonomie der Wissenschaft legt, betont T5 die Verantwortung gegenüber der Öffentlichkeit. T2 geht genau den entgegengesetzten Weg. In seinen Aussagen erscheint eine Verantwortung gegenüber der Wissenschaft, die sich nicht nur explizit von einer Leistungsorientierung gegenüber der Praxis distanziert, sondern auch die Grenzen zwischen 
Wissenschaft und Praxis klar macht. Die (Nicht-)Übernahme von Verantwortung wird also entweder in Hinblick auf die Wissenschaft oder die Praxis konzipiert. Auch hier wird auf Innen- und Außenorientierungen im Sinn von epistemischkognitiven oder sozial-politischen Selbstbeschreibungen Bezug genommen.

Wie bereits im vorhergehenden Analyseabschnitt werden hier zwei unterschiedliche Selbstbeschreibungen der Soziologie und auch Fremdbeschreibungen der Gesellschaft produziert. Im ersten Fall geht es den Interviewten darum, die Autonomie der Soziologie vor externen Leistungserwartungen der Gesellschaft zu schützen. Im zweiten Fall wiederum entsteht aufgrund der Produktion gesellschaftlich relevanten Wissens eine Verpflichtung, dieses an die „Betroffenen" weiterzugeben. Die Fremdbeschreibung, die hier über die Gesellschaft produziert wird, variiert dementsprechend: Einerseits wird sie als Bedrohung der Autonomie der Wissenschaft dargestellt, andererseits als ein - in Ulrich Becks Worten - mit soziologischem Wissen zu missionierender Korpus.

Die Argumentationsmuster, die für eine (Nicht-)Übernahme von Verantwortung genutzt werden, variieren: Während T5 überwiegend institutionell begründet hat, dass es nötig sei, Verantwortung gegenüber dem Untersuchungsgegenstand zu übernehmen, unterscheidet T6 zwischen einer persönlichen Moral und einer Moral der Wissenschaft. Er konstruiert damit einen „entmoralisierten“ Forscher und plädiert dafür, dass die Forschung keine Verantwortung gegenüber der Praxis übernimmt. T2 dagegen argumentiert im Sinne eines integren Forschenden, der externe Einflüsse und Ansprüche abwehrt, um die Autonomie der Wissenschaft zu schützen.

\subsubsection{Risiken im Umgang zwischen Wissenschaft und Praxis}

In den Interviews wurde gezielt nach Risiken, Gefahren oder Grenzen der Soziologie zu ihrem Untersuchungsgegenstand gefragt. Hier ging es darum, auszuloten, inwiefern die Interviewten explizit auf mögliche Problemstellungen zwischen Soziologie und ihrem Untersuchungsgegenstand eingehen, wie sie sie rhetorisch ausgestalten und definieren. Wir haben bereits in den vorausgegangenen Abschnitten implizit etwas über Risiken erfahren. Risiken wurden hier in Hinblick auf die Autonomie der Wissenschaft thematisiert (4.2.1) sowie bezüglich der Reichweite soziologischer Erkenntnisse, die mitunter bei den Betroffenen Schaden anrichten könnten. Der Vergleich zur Erfindung der Atombombe wurde gezogen (4.2.2). Im folgenden Abschnitt werden die Orientierungsmuster extrahiert, die explizit im Zusammenhang mit Risiken im Umgang zwischen Wissenschaft und Praxis zum Ausdruck kommen. 


\section{Analyse ausgewählter Interviewabschnitte}

\section{Interview T7, Zeile 349-395}

349

I Hm hm, ja. Ähm gibt es denn ähm bestimmte Sachen, wo Sie sagen, das sollte die Soziologie aber jetzt nicht machen, auch im Sinne von-von Engagement, Öffentlichkeit oder Transfer, also ne gewisse Grenze, wo man sagt $d a s=$

LJa ich mein' das ist wie im Journalismus, ne, man darf sich mit keiner Sache gemein machen, auch nicht mit ner guten, ne. ich äh würde sagen also äh zu starke sozusagen politische Orientierung der-der Soziologie, also ich merk' das selber, man wird auch immer relativ schnell natürlich festgelegt äh auf bestimmte Positionen und-und Haltungen, das ist durchaus äh riskant und äh äh, weil das ist für uns vielleicht interessant und wir klopfen uns dann auf die Schulter, äh aber es ist so, dass in der Öffentlichkeit äh man als Soziologe auch nicht wahrgenommen wird, wenn man immer nur wieder als ich sag's mal in Anführungsstrichen die linken Soziologen äh oder die Soziologen und Soziologinnen äh rüberkommt, sondern dass man tatsächlich irgendwie auch ein- ein Gespür für Wirklichkeit äh hat, wo- wo man sich nicht vollständig entkoppelt von Diskursen, die in der Gesellschaft oder unter Normalbürgern irgendwie auffallen, dass man für die auch interessant äh bleiben kann äh und äh ich glaub' da gibt's viele Anknüpfungspunkte und ja da seh' ich auch noch nicht jetzt nicht alles verloren, gibt ja immer mal wieder Beispiele von interessanten äh ja Büchern oder Debatten, Beiträgen, wo ich sage, ja, das äh das ist schon spannend, aber bestimmte Themen äh sind uns eben auch äh weggenommen worden. die letzten großen Bücher über Ungleichheit äh sind alle von Ökonomen geschrieben worden, //I: hm// die eben äh und das sind alles Ökonomen, also ob das jetzt Atkinson is oder Stiglitz äh oder Piketty äh sind alles Ökonomen, die auch fachlich extrem stark sind, also äh ganz wesentliche Beiträge zu ihrer Disziplin, auch durchaus quantitative Beiträge geleistet haben und trotzdem in der Lage sind, sozusagen Bücher da draufzusatteln oder Marcel Fratzscher hier-hier in Deutschland, äh und das würd' ich mir von der Soziologie im Prinzip auch äh auch erwarten, aber wir haben halt ne, sozusagen ne bisschen äh so ne bisschen- [Name der Person] ist da vielleicht die Ausnahme, aber dass häufig Leute auch sehr viel öffentlich publizieren, die jetzt nicht unbedingt die äh wissenschaftlich stärksten äh Karrieren haben und die, die wissenschaftlich sehr starke Karrieren haben, machen das halt sozusagen in der Unsichtbarkeit für die weitere Öffentlichkeit und das find ich ist schon- 
384

385

386

387

388

389

390

391

392

393

394

395

ist schon problematisch, dass da auch gar kein Anspruch mehr besteht und durchaus manchmal sogar Naserümpfen. ich mein' das Beck-Bashing äh äh ist ja Legende, ne, der hat sich da immer äh unverstanden gefühlt, natürlich ein starken Drang in die Öffentlichkeit, ist auch gehört worden, ist natürlich auch was, was die Eitelkeit durchaus auch befriedigen äh äh kann, aber ist halt in vielen Bereichen oder bei vielen Leuten in der Soziologie durchaus äh tabuisiert worden, ne und negativ gesehen worden und äh also ich sehe ihn eher wirklich als jemand, den man extrem vermissen äh äh sollte, einfach weil er ein Gespür für-für Themen, für Begriffe hatte und dass dann auch in- tatsächlich in der Öffentlichkeit lancieren konnte und ich meine, wenn's keinen Originalitätswert gehabt hätte, dann hätt's die Wissenschaft auch links liegen gelassen und das hat sie eben nich, ne.

\section{Formulierende Feininterpretation}

Thema: Distanz der Soziologie zu ihrem Untersuchungsgegenstand (Z. 349-369) T7 geht auf das Thema ein, indem er betont, dass sich die Soziologie ,,mit keiner Sache gemein machen“ darf, und den Vergleich zum Journalismus zieht. Das Risiko in einer zu starken (linken) politischen „Orientierung“ liege darin, dass man als Soziologe in der Öffentlichkeit nicht wahrgenommen wird. Er erläutert, dass die Soziologie sich weitgehend von gesellschaftlichen Diskursen „entkoppelt“ habe, wodurch es derzeit weder interessante Bücher noch Debatten gibt.

Thema: Wahrnehmung der Fachkollegen (Z. 369-384)

T7 zieht dann den Vergleich zu den Ökonomen, die in den letzten Jahren wichtige Themen in die öffentliche Debatte gebracht hätten. Diese seien in der Lage, die fachlichen und die öffentlichen Diskurse zu bedienen. In der Soziologie sieht er das anders, da gingen öffentliche und disziplinäre Anerkennung meist auseinander, was $\mathrm{T} 7$ problematisch findet.

Thema: Wahrnehmung durch die Fachkollegen (Z. 384-395)

T7 begründet dies mit einem mangelnden Interesse und einem „Naserümpfen“ innerhalb der Fachkollegenschaft. Als prominentes Beispiel nennt er Ulrich Beck („Beck-Bashing“), der in der Disziplin sehr kritisch gesehen wurde, obwohl er innerhalb der Disziplin wichtige Beiträge geleistet hat und auch in der Öffentlichkeit sehr präsent war. 


\section{Reflektierende Feininterpretation}

Thema: Distanz der Soziologie zu ihrem Untersuchungsgegenstand (Z. 349-369): Beschreibung mit dem Hintergrundmodus einer Bewertung

T7 weist auf das Risiko hin, dass die Soziologie in der Öffentlichkeit negativ wahrgenommen wird. Dies wäre dann der Fall, wenn sie wenig Distanz zur „Sache" aufwiese und Soziologen politisch linke Positionen vertreten würden. Er zieht an dieser Stelle eine Grenze zwischen der Soziologie und ihrem Untersuchungsgegenstand, und zwar in Hinblick auf die Glaubwürdigkeit der Soziologie in der Öffentlichkeit. Allerdings sieht er diese Distanzierung der Soziologie kritisch, da diese kaum noch gesellschaftspolitisch wichtige Beiträge leiste. Er kritisiert dementsprechend auch die Selbstreferenzialität der Soziologie in Hinblick auf ihre öffentlichen Beiträge.

Thema: Wahrnehmung der Fachkollegen (Z. 369-384):

Beschreibung mit dem Hintergrundmodus einer Bewertung

Der Vergleich, den T7 dann mit den Ökonomen zieht, ist aus zwei Gründen bezeichnend: Einerseits spielt T7 auf die Vereinbarkeit von wissenschaftlicher und disziplinärer Arbeit an, die er in der Soziologie nicht gegeben sieht. Er kritisiert die Fachkollegenschaft, die kein großes Interesse an dieser Vereinbarkeit zeigt. Andererseits spielt er darauf an, dass die Ökonomen den Soziologen Themen „weggenommen“ haben, die zu öffentlicher Sichtbarkeit führen. Diese Darstellung kann in Anlehnung an Gieryn als Expansion der Ökonomie in andere wissenschaftliche Domänen bezeichnet werden, denn diese beansprucht ein von der Soziologie besetztes Thema für sich: soziale Ungleichheit.

\section{Thema: Wahrnehmung durch die Fachkollegen (Z. 384-395):}

Beschreibung mit dem Hintergrundmodus einer Bewertung

T7 erklärt diese „Verdrängung“ der Soziologie durch die Ökonomie auch mit der Soziologie selbst. Die Fachkolleg(inn)en würden einer öffentlichkeitsrelevanten Soziologie mit „Naserümpfen“ begegnen. In seinen Ausführungen benutzt er weitere negativ besetzte Ausdrücke: Er stellt eine „Tabuisierung“ der Öffentlichkeitsrelevanz und ein „Bashing“ von sehr bekannten zeitgenössischen Soziologen wie Ulrich Beck fest. Obwohl er Beck einerseits auch kritisch bewertet (dieser habe möglicherweise seine Eitelkeit durch seine Öffentlichkeitsrelevanz befriedigt), nimmt er ihn als Beispiel für einen Soziologen, der wissenschaftlich relevant und öffentlich wirksam war. 
Interview T8, Zeile 308-316

308 T8 Na ja, ich bin ja in diese Debatte, es- ähm ich bin hineingekommen in diese 309 Post-68er-Phase, wo es sehr viel weltanschauliche Dimensionen in der So$310 \quad$ ziologie gab, meiner Ansicht nach sehr viel Ressentimentwissenschaft die 311 Soziologie war, //I: hm// Soziologie ist immer Ge- in Gefahr, Ressentiment312 wissenschaft zu werden. viele- viele viele Kollegen, die ich treffe, spür' ich $313 \quad$ irgendwie, die haben irgendein komisches Ressentiment und das pflegen sie 314 durch Wissenschaft also gegen Ungleichheit und irgendwie und haben was 315 gegen Reiche und irgend-dann denke ich immer, ach pfff weshalb-wie kann man nur so bl-so unreflektiert sein und so. also das treff' ich viel.

\section{Formulierende Feininterpretation}

Thema: Soziologie als Ressentimentwissenschaft (Z. 308-316)

T8 stellt die Soziologie als Ressentimentwissenschaft dar. Er führt aus, dass er auf viele Fachkollegen trifft, die ihre eigenen Wertehaltungen in die Forschung einbringen, was er nicht gut findet.

\section{Reflektierende Feininterpretation}

Thema: Soziologie als Ressentimentwissenschaft (Z. 308-316):

Beschreibung mit dem Hintergrundmodus einer Bewertung

Ähnlich wie T7 sieht auch T8 das Risiko, dass Soziologie zu normativ ist. Rhetorisch markiert er dies mit dem Begriff der „weltanschaulichen Dimension“ der Soziologie im Sinn einer „Ressentimentwissenschaft“. Auch rekurriert er auf seine Wahrnehmung der Fachkollegen, wie das bereits T7 getan hat. Er wirft ihnen vor, ihre eigenen Wertvorstellungen bzw. Ressentiments unter dem Deckmantel der Wissenschaft durchzusetzen.

Interview T10, Zeile 721-747

T10 Das hat was mit der Projektförmigkeit zu tun und hat was mit der Gefahr von Scheinpartizipation auch zu tun, dass Leute- und das gilt aber nicht nur für Communitypartner, das is schon auch, es gibt Gefahren im Prinzip für alle Personen und alle Gruppen, die mitmachen. Fang ich mal bei den Communityleuten an, die-also in dem Fall waren das ja vor allen Dingen Migrantinnen und Migranten, die kennen das ganz häufig, dass sie irgendwo an nen Tisch gebeten werden, aber eigentlich sind die Parameter schon gesetzt, und sie sollen im Prinzip nur als Dekoration dabei sein und anderen Leuten erlauben, im Prinzip $n$ schönes Bild von Multikulti oder von 
„,wir sind ja alle so interkulturell geöffnet“ "äh zu geben, ohne dass die Migranten tatsächlich gleichberechtigt am Tisch sitzen oder vielleicht auch gleichberechtigt genauso beschäftigt werden wie andere Leute, die am Tisch sitzen. [...] Und in der Wissenschaft gibts natürlich auch Gefahren, ne? also ich mach natürlich, wenn ich so ne Form der Forschung mache, wo ich mich nich nur an ein wissenschaftliches Publikum richte, sondern eben auch an die Allgemeinheit oder an marginalisierte Gruppen und die Praxis, bin ich natürlich auf der einen Seite in der Wissenschaft, steh ich son bisschen dubios da, ich mein der der Ansatz der Aktionsforschung is ja hier einfach auch so besonders schräg verlaufen, anders als in anderen Ländern, wo man eigentlich gar kein akademisches Standing hat damit, also da arbeiten wir gerade daran, dass der Ansatz wieder $n$ bisschen stärker auch äh legitimiert wird, und besser angesehen und besser gefördert wird. aber das is für mich auch-warn Riesenrisiko, ja? wo ich auch- hätt ich geforscht, hätt ich ganz anders das akademische Publikum bedienen können und hätte jetzt auch vielleicht andere Chancen im Arbeitsmarkt, keine Ahnung, also das kann man im Nachhinein immer schlecht sagen, aber die Gefahren sind in jedem Fall da.

\section{Formulierende Feininterpretation}

Thema: Gefahr für die Praxis (Z. 721-733)

T10 beschreibt am Beispiel eines Forschungsprojekts, dass es bei einer Zusammenarbeit zwischen Wissenschaft und Praxis Gefahren für die beteiligten Gruppen gibt. Für die „Communityleute“ besteht die Gefahr, dass sie nur scheinbar partizipieren.

\section{Thema: Gefahr für die Wissenschaft (Z. 733-747)}

In diesem Abschnitt beschreibt T10 die Gefahren für die Wissenschaft. Er nennt einerseits die kritische Haltung in der Wissenschaft einer solchen Arbeit gegenüber, andererseits die geringeren Karrierechancen, die man innerhalb der Wissenschaft hat, wenn man solche Projekte betreibt.

\section{Reflektierende Feininterpretation}

Thema: Gefahr für die Praxis (Z. 721-733):

\section{Beschreibung mit dem Hintergrundmodus einer Bewertung}

T10 geht zunächst auf die „Gefahren“ für die an einem Forschungsprojekt beteiligten Praxispartner ein. Er konzipiert den Begriff der Gefahr in zwei Richtungen, wobei eine Grenzziehung zwischen einer nicht-wissenschaftlichen Community (Communitypartner) und der Wissenschaft selbst zutage tritt. Die Gefahren 
für die Communitypartner thematisiert T10 als Hierarchiegefälle, das zwischen Migrant(inn)en und anderen Personen entsteht, die an dem Prozess beteiligt sind. Er bezeichnet dies als „Scheinpartizipation“ bzw. „Dekoration“. Hier wird deutlich, dass T10 ein Bild des Austauschs zwischen Wissenschaft und NichtWissenschaft zeichnet, das deliberativen Prinzipien entspricht und in dem die Praxis nicht einfach als Wissenszulieferer gesehen wird.

Thema: Gefahr für die Wissenschaft (Z. 733-747):

Beschreibung mit dem Hintergrundmodus einer Bewertung

T10 konzipiert ebenso „Gefahren“ für die Wissenschaft. Ähnlich wie bei T7 bestehen diese aufgrund der mangelnden Akzeptanz der Fachkollegen. T10 beschreibt, dass er als „dubios“, also nicht vertrauenswürdig wahrgenommen wird, wenn er eine öffentlichkeitswirksame Soziologie betreibt. Dies ist eng mit seinem spezifischen (praxisnahen) Forschungsansatz verbunden, der in der deutschen Soziologie bislang keine Rolle spielt. Eng damit verknüpft ist die Gefahr für die wissenschaftliche Karriere: Das sogenannte Bedienen eines wissenschaftlichen Publikums war mit diesem Ansatz nur begrenzt möglich. Es passt also nicht in die Konventionen der Disziplin und ist damit ein Nachteil für die persönliche Karriereentwicklung. Die Gefahren für die Wissenschaft werden also strategisch interpretiert und nicht in Hinblick auf die wissenschaftliche Autonomie.

Interview T1, Zeile 631-652

631 T1 Die Risiken liegen finde ich ja auf der Hand. also erstens wird ja an wissenschaftliche Akteure der Anspruch eines gewissen Reflexionsgrads und Komplexitätsgrads auch also also als Anspruch gestellt und den kann man natürlich nicht hemmungslos unterbieten, ja, das ist klar. und das ist natürlich ne Gratwanderung, also da äh das richtige äh die richtige Abstraktionshöhe zu finden und Konkretisierungsgrad oder hm, ist einfach schon schwierig, ne. aber ich glaube, grade wenn mans nicht als als Individuum macht, sondern wenns ne Organisation macht, dann kann ja auch stärker gemeinsam reflektieren, also wo jetzt sozusagen der Grat is äh, den man noch begehen kann und wo man runterfällt, ja. aber das ist glaub ich-es is klar, es is- gerechtfertigterweise gibt es bestimmte Ansprüche an eine wissenschaftliche Intervention, die andere sind als an eine politische, ja? das is klar, ahm, das find ich ne ne große Herausforderung ahm und ich finde, aber also auf individueller Ebene is es natürlich einfach die Herausforderung, die Herausforderung is die Ressourcen, ja? man hat einfach zu wenig Zeit. ich meine, das 
646

647

648

649

650

652

sagen Ihnen ja die NachwuchswissenschaftlerInnen ja auch, ja, ich würde gerne nicht nur den einen Weg bedienen, aber damit ich bin ich hier schon völlig ausgelastet. und wenn ich mich hier sehe als durchschnittlichen äh Hochschullehrer einer durchschnittlichen deutschen Universität, womit ich meine Zeit verbringe, und wie viel unterschiedlichste Dinge von mir erwartet werden, und ich erwarte von mir selber noch ein paar darüber hinaus, dann bleibt natürlich auch einfach wenig Zeit.

\section{Formulierende Feininterpretation}

Thema: Risiken auf Ebene der Wissenschaft (Z. 631-643)

T1 definiert Risiken in Hinblick auf den „Anspruch eines gewissen Reflexionsgrads und Komplexitätsgrads“ an die Wissenschaft. Er stellt die Erfüllung dieses Anspruchs und das Angebot des ,richtigen Konkretisierungsgrads“ als „Gratwanderung" dar. Hier rekurriert er auf eine Lösungssuche auf organisationaler Ebene.

\section{Thema: Risiken auf individueller Ebene (Z. 643-652)}

Im Folgenden definiert T1 Risiken auf der individuellen Ebene, die sich insbesondere auf die mangelnden Ressourcen als Herausforderung beziehen. Diese sieht er einerseits bei Nachwuchswissenschaftler(inne)n, andererseits bei sich selbst.

\section{Reflektierende Feininterpretation}

Thema: Risiken auf Ebene der Wissenschaft (Z. 631-643):

\section{Argumentation mit dem Hintergrundmodus einer Bewertung}

In seiner Argumentation stellt T1 zwei Aspekte einander als „Gratwanderung“ gegenüber, die für den Anspruch an wissenschaftliches Arbeiten wie an ,eine politische Intervention“ gelten. Als „wissenschaftlich“ beschreibt er einen gewissen Reflexions- und Komplexitätsgrad bzw. eine Abstraktionshöhe, die er im weiteren Text als „Ansprüche an eine wissenschaftliche Intervention“ markiert. In einer ,politischen Intervention“ ist der angemessene Grad an Konkretisierung wichtig. Die wissenschaftliche und politische Intervention sieht er als schwer miteinander vereinbar und bezeichnet dies als Herausforderung. Diese stilisiert T1 als Gefahr, indem er klar macht, dass dieser Grat nicht nur schwierig zu begehen ist, sondern auch einer ist, „wo man runterfällt“. Es gibt also Grenzen, die nicht überschritten werden dürfen. Seine Lösung ist eine gemeinsame Reflexion auf organisationaler Ebene. Die individuelle Herausforderung soll auf kollektiver Ebene gemeistert werden, womit jedoch nicht die Disziplin gemeint ist, sondern die Organisation. 
Thema: Risiken auf individueller Ebene (Z. 643-652):

Beschreibung mit dem Hintergrundmodus einer Bewertung

Diese Argumentation setzt T1 nun auf individueller Ebene fort: Die Herausforderung sind insbesondere die zeitlichen Ressourcen. Er geht in die Defensive: T1 würde gerne ,nicht nur den einen Weg bedienen“, was ihm jedoch aufgrund seiner vielfältigen Verpflichtungen als Hochschullehrer kaum möglich ist. Auch hier argumentiert er mit unterschiedlichen Anforderungen an eine wissenschaftliche und politische Intervention. Herausforderungen auf individueller Ebene werden wiederum als institutionelle Herausforderungen in Hinblick auf diverse Verpflichtungen thematisiert, die von außen an T1 gestellt werden.

\section{Orientierungsmuster „Risiken im Umgang zwischen Wissenschaft und Praxis“"}

\section{Inadäquate Grenzziehung zwischen Soziologie und Nicht-Soziologie}

Die Risiken im Umgang zwischen Wissenschaft und Praxis wurden in inadäquaten Grenzziehungen zwischen Soziologie und Nicht Soziologie gesehen.

Die Gefahr wird einerseits in einer zu geringen, andererseits in einer zu rigorosen Grenzziehung zwischen Soziologie und Nicht-Soziologie gesehen. Die Gefahr einer zu geringen Grenzziehung ging letztlich auf die Doppelstruktur der Soziologie zurück, die sich darin äußert, dass man sich zu wenig vom Gegenstand distanziert. Die Selbstbeschreibung, die dem inhärent ist, ist die des parteinehmenden Forschers, der sich mit dem Gegenstand verbrüdert (T7) oder einer „Ressentimentwissenschaft“ (T8). Diese Art von Selbstbeschreibung beinhaltet zudem eine Fremdbeschreibung der Gesellschaft, die die persönlichen und politischen Interessen der Forschenden spiegelt. T1 sieht die Schwierigkeit, eine „wissenschaftliche Intervention“ mit einer „politischen Intervention“ zu verbinden, da an diese Arten von Interventionen unterschiedliche Kriterien angelegt werden. Er rekurriert insbesondere auf die Erfüllung wissenschaftlicher Standards und auf die „Gefahr“, wenn man ihnen nicht genügt.

Auf der Seite einer zu starken Grenzziehung zum Untersuchungsgegenstand steht wiederum die Selbstbeschreibung, dass die Soziologie Gefahr läuft, den Kontakt zu „realweltlichen Themen“ zu verlieren. Hier wird eine Fremdbeschreibung der Gesellschaft aktiviert, in der sich die Soziologie als die „bessere“ Form von Wissen geriert, was letztlich zu einem unausgeglichenen Verhältnis zwischen der Soziologie und ihrem Untersuchungsgegenstand (Scheinpartizipation) führt. Die Selbstbeschreibung, die T10 damit produziert, ist eine, die die hierarchischen Grenzen zwischen der Soziologie und ihrem Gegenstand auflöst. 
Im Zentrum dieser Selbstbeschreibungen steht letztlich die Angst vor einer inadäquaten Grenzziehung zum Untersuchungsgegenstand.

\section{Ausschluss durch die „invisible colleges“}

Ein Orientierungsmuster, das in Hinblick auf die Risiken im Umgang zwischen Wissenschaft und Praxis eine hervorgehobene Rolle spielte, ist die Wahrnehmung durch die Peers. Meist werden keine Fachkollegen beim Namen genannt, es ist vielmehr von „,invisible colleges“ die Rede, die nicht näher spezifiziert, aber doch im Sinne einer „Fachöffentlichkeit“ wahrgenommen werden. Bereits T10 hat in seiner Antwort hervorgehoben, dass eine mangelnde Grenzziehung zwischen Soziologie und Nicht-Soziologie in der Fachkollegenschaft als ,dubios“ angesehen wird und wenig Vertrauen weckt. Verstärkt wird dies durch die Anwendung eines Forschungsansatzes, der innerhalb der (deutschen) Community wenig akzeptiert ist. Auch T7 stellt heraus, dass ein Engagement in der Öffentlichkeit „Naserümpfen“ hervorrufen kann. Er nimmt als Beispiel einen prominenten Soziologen, nach dem Motto „Wenn es ihm nicht gelungen ist, wem kann es dann gelingen?“.

Auch T1 äußert sich an einer Stelle zu der Wahrnehmung durch die Fachkollegen:

„Wer sich eher soner Öffentlichkeitswirksamkeit verschreibt, aus welchen Gründen auch immer ja? Geltungsbedürfnis äh weiß nicht, dass man vielleicht auch Geld für kriegt oder ah egal, is ja häufig eher schlecht beleumundet in der Disziplin, ja? also wir wissen alle irgendwie, die bekanntesten deutschen Soziologen, [Name der Person] oder [Name der Person], werden gleichzeitig irgendwie jetzt nicht belächelt, aber schon auch kritisch gesehen, ja, dass die sich dann halt weit ausm Fenster lehnen und man kann ja von dem, was sie inhaltlich sagen, halten, was man will ja? aber überhaupt, die Tatsache, dass die dann irgendwie in Hochglanzjournalen oder- nun da kann man sagen, gut das is- da isn Neidaspekt mit drin, is aber egal, ich glaube erst einmal is es $n$ schwieriger Rollenwechsel oder ne Rollenkombination, wissenschaftlich tätig zu sein und das is- ich meine, beide sind ja eigentlich hochkarätige Wissenschaftler, und gleichzeitig aber den Anspruch zu haben, so was so zu vermitteln, so dass es nicht nur WissenschaftlerInnen verstehen. ahm und dann kommt dieser dieser mehr öffentlichkeitsbezogenen oder vielleicht auch transferbezogenen- jetzt in dem Sinn, wie ich ihn verstehen würde- ähm transferbezogenen Soziologie auch immer ein bisschen Naserümpfen jetzt aus dieser Wertfreiheits- Werturteilsstreitsperspektive zu, ne? also dass man sagt, also wir können nichts sagen, wir können uns nicht positionieren, wir haben kein autoritatives Wissen, wir haben auch keine ja höhere Position, die wir einnehmen können gegenüber ahm jedem anderem Mitglied in dieser Gesellschaft, das würde ich alles teilen, ja, und deswegen müssen wir uns zurückhalten. und 
deswegen gibt es glaube ich schon ähm- naja aber, ich würds umgekehrt sehen, also ich würde, sozusagen, die Soziologie, die $\underline{\text { ich }}$ betreibe, als die reputierlichere, ja, gesellschaftlich sehen als die andere, also wenn man jetzt mal von Lagern sprechen möchte. aber es ist klar, dass man mit bestimmten Sachen in die Kölner Zeitschrift kommt, mit anderen nich, ja? und dann aber, wenn man nich in die Kölner Zeitschrift kommt, aber vielleicht eher mal ins Zeit-Feuilleton oder Ähnliches, is klar. ahm, aber ansonsten gibt es glaube ich schon so ne Reputationshierarchie in dem Sinne, dass man, wenn man sich auf die Wissenschaftlichkeit im engeren Sinne konzentriert, dass das(2) alle anderen Dinge erstmal gleich- dass das schon das Reputierlichere ist. und ich glaube, man muss besondere sozusagen Qualitätsanforderungen erfüllen, wenn man das andere auch noch macht. [Name der Person] und [Name der Person] können sich das leisten und werden trotzdem glaube ich als Wissenschaftler ernst genommen, wenn man nur eine Stufe darunter ist, ist man sozusagen für die Fachöffentlichkeit gestorben. [...] aber wenn man jetzt sozusagen nicht gleichzeitig irgendwie an den- also die Standards, die die Disziplin setzt, ja, an Publikationen, Drittmitteln oder Ähnlichem, wenn man die nicht gleichzeitig erfüllt, hat man es ganz ganz schwer, ahm, dann irgendwie diese Öffentlichkeitsschiene zu bedienen, ahm, weil man dann irgendwie den Halt verliert in der in der Akademie selbst.“ (T1, Z. 337357)

Auch in diesem Zitat zeigt sich deutlich, inwiefern eine Fremdbeschreibung der „,invisible colleges“ (Crane, 1972) maßgeblich für die Konstruktion des Verhältnisses der Soziologie zu ihrem Untersuchungsgegenstand ist. T1 bringt einen Vergleich, in dem sogar hoch reputierte Fachkollegen einen schwierigen Stand innerhalb der Community haben. Jedoch nur „eine Stufe darunter“ sei man für die „Fachöffentlichkeit gestorben“, wenn man Wissenschaft allgemein verständlich macht. In seiner Argumentation bezieht sich T1 insbesondere auf normative Aspekte der Forschung und den Diskurs der „Werturteilsstreitperspektive“ als Referenz für das wissenschaftliche „Lager“. T1 nimmt eine Grenzziehung in Hinblick auf die Autorität wissenschaftlichen Wissens gegenüber anderen Wissensarten vor: „Wir können nichts sagen, wir können uns nicht positionieren, wir haben kein autoritatives Wissen, wir haben auch keine höhere Position [...] und deshalb müssen wir uns zurückhalten.“

Ein Risiko in Hinblick auf den Umgang zwischen Wissenschaft und Praxis ist also in der Selbstbeschreibung auch die Wahrnehmung der ,invisible colleges“. Eine Abgrenzung zum Untersuchungsgegenstand findet in dieser Hinsicht also nicht über den Untersuchungsgegenstand selbst statt, sondern über die Wahrnehmung der wissenschaftlichen Community. Das verschiebt den Grenzdiskurs auf einen internen Grenzdiskurs, die Teilnahme an der Community scheint prekär und auch bei großen wissenschaftlichen Leistungen nicht gesi- 
chert. Vielmehr folgt die Selbstbeschreibung der Sorge eines möglichen Ausschlusses durch die Community.

\subsection{ZWISCHENFAZIT}

\subsubsection{Das Verhältnis zur Soziologie als fragile Identitätskonstruktion der Selbstbeschreibung}

In der Analyse des Verhältnisses von Soziolog(inn)en zur Soziologie selbst (Abschnitt 4.1) konnten wir sehen, dass es diverse Selbstbeschreibungen auf unterschiedlichen Ebenen gab. Es zeigt sich, dass die Soziologie über keinen konsolidierten Kern verfügt, der ähnliche Selbstbeschreibungen zur Verfügung stellt. Vielmehr zeichnet sich die Soziologie durch eine Interpretationsoffenheit aus, die mit unterschiedlichen Sinnkonstruktionen gefüllt wird. Bezeichnend war zudem, dass keiner der Interviewten äußerte, er habe von Anfang an die Absicht gehabt, Soziologie zu studieren. Selbstbeschreibungen wurden mit diversen biografischen Erfahrungen, Enttäuschungen oder Erwartungen angereichert und die Motivation für Soziologie wurde unterschiedlich begründet. Letztlich konnten zwei immer wiederkehrende Charakteristika der Selbstbeschreibung ausgemacht werden: die Orientierung an epistemisch-kognitiven bzw. an sozial-politischen Kriterien der Sinnkonstruktion. Epistemisch-kognitive Kriterien orientierten sich stärker an einer Selbstbeschreibung der „Soziologie als Wissenschaft“, während eine Ausrichtung an sozial-politischen Kriterien sich an einer „Soziologie als Veränderung der Gesellschaft" orientierte. Bereits im Verhältnis der Interviewten zur Disziplin selbst zeigte sich also, dass die Doppelstruktur der Soziologie, ihre Einbindung in den eigenen Gegenstand, bedeutungsvoll für die Selbstbeschreibungen war. Insbesondere kam dies in den Selbstbeschreibungen zur Frage der Wertneutralität der Soziologie zum Vorschein, und ebenso in der Frage zur Motivation für die Soziologie. In beiden Fällen waren ähnliche Muster der Exklusion vs. Inklusion zu beobachten.

Eine Selbstbeschreibung, die sich an sozial-politischen Kriterien orientierte, war mitunter mit einer Legitimierungsrhetorik verbunden. Ausschlaggebend war dafür, inwieweit sich der oder die Interviewte in einem „Innen“ oder „Außen“ der Disziplin verortete. Die Rolle disziplinärer Referenzfolien gewann in Hinblick auf den Umgang mit Werten in der Soziologie einen besonderen Stellenwert. Auch diese Referenzfolien, beispielsweise disziplinäre Vertreter wie Max Weber oder Theorierichtungen wie die kritische Theorie, dienten zur Legitimie- 